\title{
The Recording and Quantification of Event-Related Potentials: II. Signal Processing and Analysis
}

\author{
Paniz Tavakoli, a, Kenneth Campbell a ${ }^{\mathrm{a}}$ \\ a School of Psychology, University of Ottawa
}

\begin{abstract}
Event-related potentials are an informative method for measuring the extent of information processing in the brain The voltage deflections in an ERP waveform reflect the processing of sensory information as well as higher-level processing that involves selective attention, memory, semantic comprehension, and other types of cognitive activity. ERPs provide a non-invasive method of studying, with exceptional temporal resolution, cognitive processes in the human brain. ERPs are extracted from scalprecorded electroencephalography by a series of signal processing steps. The present tutorial will highlight several of the analysis techniques required to obtain event-related potentials. Some methodological issues that may be encountered will also be discussed.
\end{abstract}

Keywords " Event-related potentials, signal processing, quantification, cognitive processes

$\equiv$ kenneth.campbel@uottawa.ca

\section{Introduction}

Event-related potentials (ERPs) are the minute changes in the electrical activity of the brain that are elicited by a physical stimulus or a psychological event. The first article in this series (Tavakoli \& Campbell, 2015) discussed the need for careful control over the physical characteristics of the stimulus but also the need to monitor psychological events - what the subject is doing. One of the major reasons for this is that there is little the researcher can do to correct errors in the design of the study, once the data have been collected. It is not possible to correct for poor stimulus-response control in an off-line manner. This is not the case for the collection of the EEG data. While care must also be taken to assure high quality acquisition of the EEG, as this second article will discuss, it is possible to clean and correct "noisy" EEG data. Still, as emphasized in the first article, prevention is better than cure. A series of steps should be employed to assure a high quality EEG signal. Nevertheless, even with exceedingly high quality data, the ERP "signal" will always be much smaller than the background "noise" of the EEG.

Several methods exist for extracting the ERP signal from the background electroencephalogram (EEG) noise. The most powerful means to do so is through the use of signal averaging (Dawson, 1954). Averaging works best when the amount of noise is more or less constant from one stimulus repetition to the next. Prior to averaging, a series of steps can be employed to "clean up" the raw EEG data in an attempt to reduce the amplitude of the noise. There are two major sources of artifact within the EEG. The first is caused by electromagnetic fields around the subject such as power lines and electric transformers or motors. During the original recording of the EEG, these can be reduced substantially by placing the subject as far away from these sources of artifact as possible, grounding the subject and ensuring interelectrode impedance is low. The second major source of artifact is a result of the picking up of extraneous electrophysiological signals from the subject, for example, the electrocardiogram, skin potentials ("GSR"), muscle movement and most importantly, eye movement artifact. A good deal of this artifact can be reduced by proper electrode application and instructions to the subject to reduce movement and to fixate the eyes on a fixed point. Nevertheless, in spite of the best attempts, subjects will still move and blink. The present article will discuss the necessary steps required for the extraction of the ERP from the noisy background of the EEG and the eventual analyses and quantification of the ERPs. Methodological issues encountered throughout this process will also be discussed.

Very powerful commercial software exists for the analysis of EEG/ERP data (e.g. Brain Products, 
NeuroScan). Each of these commercial programs offers different general-purpose options for data quantification. Some researchers do, however, choose to write their own ERP analysis program often using open source software (e.g., EEGLAB, ERPLAB). These have the obvious advantage of being low cost, providing many of the same quantification procedures available in the commercial systems, albeit perhaps not as "user friendly". They also allow the researcher to write their own software for analyses that are not available in the general-purpose commercial systems. The choice of which software to use largely depends on what type of analyses are being carried out. Signal processing, artifact correction and scoring methods that are used in most ERP labs will be discussed here.

\section{Reduction and Correction of Noise}

\section{Filters}

Many of the sources of artifact can be largely attenuated through the judicious use of filters, providing the artifact contains frequencies that are lower or higher than those of the actual ERPs of interest. Two stages of filtering can be applied; initial real-time analog filters used for the recording of the EEG and subsequently, offline filters. Both types of filters provide for a low filter (attenuating low frequencies but allowing higher frequencies to pass) and a high filter(attenuating high frequencies but allowing low frequencies to pass). Because a low filter allows higher frequencies to pass while the high filter allows for lower frequencies to pass, they are also called high- and low-pass filters respectively.

With older EEG systems, analogue filters were typically applied to the data by effectively adjusting the resistor and capacitor circuitry in the amplifier. Such analog filters are applied in real-time during data acquisition (within the EEG amplifiers), before the signals are converted to digital format. Analog filters are however imperfect and can shift the latency of signals causing distortions in the data. With the advent of modern, rapid computers, digital filtering is now the preferred method of choice, eliminating the need for precise analog filtering. For this reason, a very broad range of frequencies is typically allowed to pass unfiltered through the analog system. The EEG signals can then be filtered more precisely off-line using digital filtering techniques.

Digital filters can be designed to avoid latency shifts in the signal. Because the digital filter is applied after data collection, its effects can be later removed and different filter parameters can subsequently be employed. They can operate in either the time or frequency domain. For example, a very simple time domain filter can be created using a smoothing or "moving average" algorithm. Each data point is replaced by the average of " $n$ " data points. For example, in a 3point moving average, the second data point might be replaced by the average of the first, second and third data points; the third data point would be replaced by the average of the second, third and fourth data points. Again, with the advent of powerful and low cost computers, much more sophisticated time-based algorithms are now available. Digital filters can also operate in the more computational-demanding frequency domain. Often, the frequency spectrum of the EEG signal is computed using a Fourier transform. This algorithm would allow for different weightings for each of the frequencies within a signal. The EEG signal is then filtered by applying an inverse Fourier transform.

Many other different digital filters can be designed, using different mathematical computations. Common to all digital filters is what is called the transfer function and it is the mathematical modelling of the filter that describes how a given input will be attenuated. There are now several different digital filters that are available and each may affect the EEG in some way, although these changes are often subtle. The computational bases for many of these digital filters are beyond the scope of the present article. Several other articles are however available that provide a detailed account of different filtering procedures and their effects on the EEG data(Cook \& Miller, 1992; Luck, 2014; Picton, Lins, \& Scherg, 1995, Picton, 2011).

Artifact containing frequencies above or below those of interest can be attenuated through the use of high and low filters, respectively. A separate "notch" filter is often used to attenuate line frequencies (50 or $60 \mathrm{~Hz}$ ). The cut-off frequency is the frequency at which the filtered power (can be considered as the area under the curve) is reduced by half $(-3 \mathrm{~dB})$. This corresponds to a $71 \%$ reduction in amplitude of the EEG. Most ERP components in cognitive neuroscience studies consist of frequencies between 0.01 and $30 \mathrm{~Hz}$. Often a high filter between 20 to $40 \mathrm{~Hz}$ is thus applied to cognitive ERP data. The use of a high filter is illustrated in the left portion of Figure 1. The ERP in this figure is contaminated by muscle artifact (frequencies above $100 \mathrm{~Hz}$ ) and $60 \mathrm{~Hz}$ line frequency. Using a high frequency cut-off below $40 \mathrm{~Hz}$ will largely attenuate 


\section{Effects of Filtering}
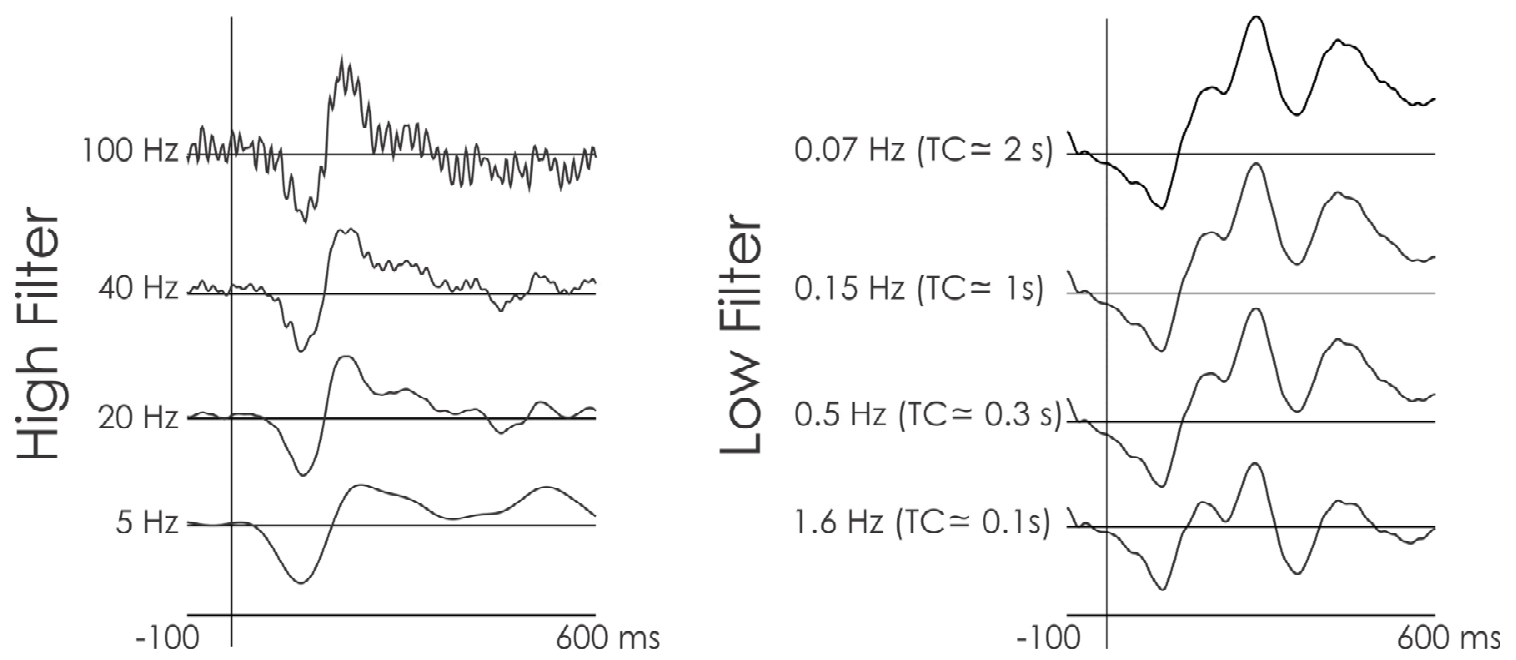

Figure 1 - Effects of filtering. An averaged ERP was computed beginning 100 ms prior to the onset of a stimulus and continuing for $600 \mathrm{~ms}$ following it. Different high and low filter cutoffs were applied to the raw EEG prior to averaging. In the left portion of the Figure, the effects of high filtering are illustrated. The ERP contains a large amount of high frequency noise after a $100 \mathrm{~Hz}$ filter is applied. Much of this is $60 \mathrm{~Hz}$ activity comes from the mains power. This is much attenuated with a $40 \mathrm{~Hz}$ high filter cutoff. A $20 \mathrm{~Hz}$ high filter further attenuates most of the remaining noise. A $5 \mathrm{~Hz}$ filter however distorts the actual ERP frequencies. In the right portion of the Figure, an ERP that contains a large amount of "drift" is illustrated. This can be attenuated by reducing the time constant from 1 to $0.3 \mathrm{~s}$. The use of a $0.3 \mathrm{~s}$ time constant may also however distort the true ERP. This is especially apparent when a $0.1 \mathrm{~s}$ time constant is employed. Thus, the use of increasingly extreme high cut-off frequencies does result in a "smoother" but more distorted ERP waveform. Similarly very short time constants will remove much of the slow drift in the ERP but may be attenuating the true ERP waveform. Caution must be taken to choose the most appropriate filter setting without severely distorting the ERP response of interest.

these sources of artifact. In addition, the raw EEG may appear to slowly "drift" over a period of seconds, appearing as a slow waxing and waning of waveforms. This is often a result of skin potential artifact caused by perspiration. Changes in electrode impedance can also lead to slow drifts in the EEG signal. A low filter of about $0.3 \mathrm{~Hz}$ is often employed in clinical EEG settings to remove this slow drift. In the cognitive laboratory, it is not unusual to observe drifts in the ERP signal associated with long-lasting and slow mental processes (i.e., actual brain activity). A $0.3 \mathrm{~Hz}$ low filter might thus inadvertently remove a true brain signal of interest. In such cases, a $0.01 \mathrm{~Hz}$ low filter might be applied. It is usually easier to understand the low filter cut-off as a "time constant", the time required for a slow frequency to be reduced by $50 \%$ of its power. A $0.3 \mathrm{~Hz}$ low filter corresponds to a time constant of about $0.5 \mathrm{~s}$. Slow ERP potentials are often observed when the subject develops a expectancy for an upcoming stimulus. For example, if the subject is presented with two stimuli, S1 and S2, the time between them being $3 \mathrm{~s}$, a slow negative potential will develop between the onset of S1 and the onset S2 if the subject is asked to prepare to respond to $\mathrm{S} 2$. This slow negative potential will therefore last about $3 \mathrm{~s}$. If the $0.3 \mathrm{~Hz}$ low filter is used, it would much attenuate the true ERP negative slow potential. To avoid this, a time constant of perhaps $6 \mathrm{~s}$ (about $0.02 \mathrm{~Hz}$ ) or more might be employed. Thus, a slowing drifting EEG would be attenuated by $50 \%$ of its power (again, $71 \%$ of its amplitude) after $6 \mathrm{~s}$. This would thus allow a $3 \mathrm{~s}$ negative slow wave to pass unattenuated. The use of a low frequency filter is illustrated in the right portion of Figure 1.

Although filters can be a great asset in increasing the signal-to-noise ratio, they can also severely distort ERP components. This is particularly the case when the frequency range of the noise and the true ERP signal overlap. As is apparent in Figure 1, the data becomes much smoother with severe high filtering (for example, when a $5 \mathrm{~Hz}$ high filter was applied). This may cause researchers to believe that the heavier the filter, the cleaner the data. Filtering will always distort the true 
EEG signal, whether this is noticeable with visual inspection or not. In Figure 1, the progressively lower cut-off frequencies for the high filter have caused the amplitude of the positivity at about $300 \mathrm{~ms}$ (the "P3" component) to become attenuated. Similarly, the higher cutoff values for the time constant have caused the entire waveform to be shifted in a negative direction. Filters can also change the onset or duration of ERP components, turn monophasic waveforms into multiphasic ones, and interfere with source localization techniques (Cook \& Miller, 1992; Luck, 2005). Luck (2005) describes this distortion in filtering as "precision in the time domain is inversely related to precision in the frequency domain" (p. 182). The more heavily the data are filtered, the more distorted the signal will become. Filtering can result in considerable distortions in the latency and amplitude of a signal and, therefore, should be used with great caution (see Luck, 2014; VanRullen 2011, Widmann \& Schroger, 2012 for detailed accounts). In most situations, some amount of filtering will be necessary to appropriately interpret the EEG/ERP data. In these instances, it is essential to know and understand the effects of filtering on the data and to carefully adjust filter-cutoff frequencies for an optimal balance between suppression of noise and distortion of the ERP waveform.

\section{Ocular correction}

Certain types of artifact will share the frequency characteristics of a true EEG signal. If filtering is used to attenuate these artifacts, it will also attenuate the true ERP signal. Other methods can be used to remove these sources of artifact. Signal averaging techniques (discussed later) will attenuate noise that is not timelocked to the stimulus. The heart beat is a low frequency source of artifact that may share the same frequency spectra as the ERP. Filtering, hence, cannot be used to remove this source of artifact. The heart beat is however not timelocked to the stimulus or response. Because it occurs at random times with respect to the stimulus, signal averaging techniques will attenuate it, providing that sufficiently large number of stimuli are presented.

The most prevalent types of artifact occurring in the EEG are blinks and eye movements. The electrical potentials created by these sources may be much larger than the EEG signal and can propagate across much of the scalp. The artifact is especially large near the eyes and over frontal regions of the scalp. To avoid these sources of artifact, subjects should be provided with a fixation point and instructed to focus on it during the experimental task and to avoid blinking as much as possible. Inevitably, however, the EEG will be contaminated by at least some eye movement and blink artifact. The frequency spectrum of eye movement artifact does overlap partially with the actual EEG and therefore filtering cannot be applied. Signal averaging techniques are also problematic because the eye movements may be time-locked to either the stimulus or the response. A subject might, for example, occasionally blink immediately following a highly relevant stimulus and less often following stimuli that are not relevant. Thus, the blink occurs at predictable times after stimulus onset and is not a truly random event (i.e., it is time-locked to the stimulus). In these cases, the researcher may misinterpret the much larger apparent ERP over the frontal regions of the cortex following the presentation of the very relevant compared to the less relevant stimulus as reflecting activation of the frontal lobe rather than true eye movement (EOG: electrooculogram) artifact

Various methods exist for the rejection and/or correction of ocular artifacts within the EEG. A common, older method is to reject any trial in which eye movement artifact exceeds an amplitude voltage that would be considered to be abnormal for the EEG. Eye blinks will typically exceed $100 \mu \mathrm{V}$ but an EEG signal this large would most likely be considered abnormal(the waking EEG rarely exceeds $100 \mu \mathrm{V}$ ). Thus all data in any trial in which the EOG exceeds $+100 \mu \mathrm{V}$ could be rejected from further analyses. Some blink artifacts may be missed if they do not exceed this predetermined threshold and for this reason, other researchers lower this threshold to a more conservative $+75 \mu \mathrm{V}$ or even $+50 \mu \mathrm{V}$. Relying on this more conservative threshold will, however, result in increased numbers of trials being rejected because of artifact contamination. The artifact rejection method is quite effective if the amount of artifact is limited. In cases of young children, the elderly and different patient populations, a very large number of blinks and movements may occur. If the rejection method is used, much of the data might therefore be rejected.

EOG correction, rather than rejection, methods have now been developed to avoid a large loss of data. Several ocular correction techniques have been developed for this particular purpose, some operating in the time domain (e.g., Gratton, Coles \& Donchin, 1983) while others also in the frequency domain (e.g., Woestenburg, Verbaten, \& Slangen, 1983). Common to 


\section{ICA Ocular Artifact Correction}

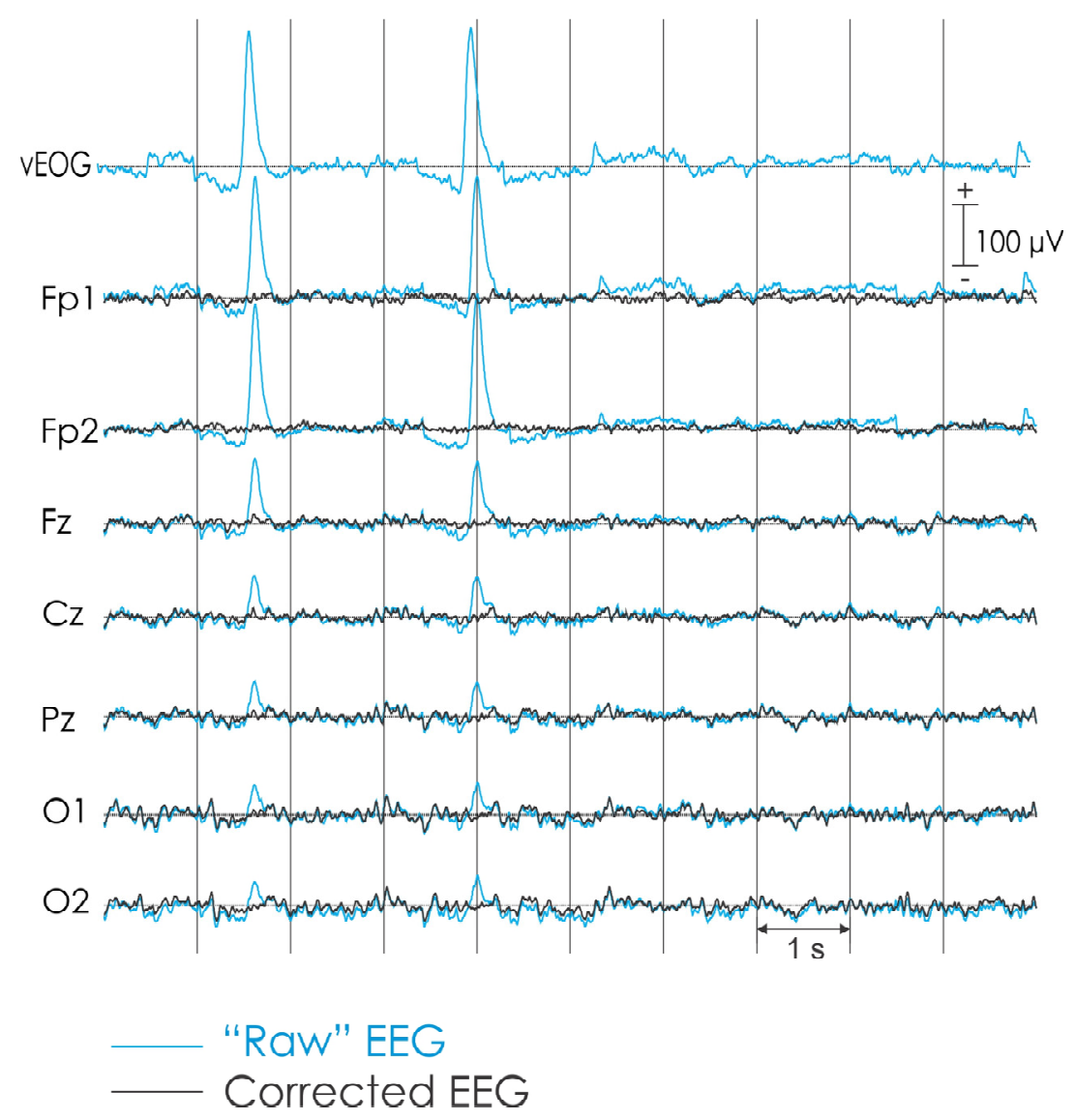

Figure 2 - Independent component analysis (ICA) ocular artifact correction. A 10 second segment of continuous EEG data are displayed. The EOG activity is displayed on the first line (vEOG: vertical electrooculogram). As is apparent, two very large upward deflections (greater than $+300 \mu \mathrm{V}$ ) are apparent. These are eye blinks. The uncorrected EEG is displayed in blue. The blink artifact is propagated to a much greater extent over anterior than posterior regions of the scalp. An ICA algorithm was applied to the data. It identified eye blinks and then partialled out this artifact. The corrected EEG is shown in black.

all correction methods is the use of mathematical/statistical algorithms to subtract or partial out the EOG activity within the EEG. The EOG artifact is considerably larger over anterior than over posterior sites. EOG correction methods thus calculate the propagation factor of the EOG signal across all areas

of the scalp, and subtract this proportion of activity from the EEG at each corresponding electrode site. Another method employs source localisation modelling to identify the sources of the artifact (EOG activity) independent of true brain source activity (Lins, Picton, Berg, \& Scherg, 1993a; 1993b). A more recent technique employs independent component analysis (ICA; Jung, Makeig, Humphries, Lee, McKeown, Iragui, \& Sejnowski, 2000; Jung, Makeig, Westerfield, Townsend, Courchesne, \& Sejnowski, 2000). ICA aims to decompose data into statistically independent components utilizing higher-order statistical measures (beyond those used by principal components analysis; PCA). These methods represent a subclass of the blind source separation (BSS) algorithms (Belouchrani, Abed-Meraim, Cardoso, \& Moulines, 1997; Joyce, Gorodnitsky, Kutas, 2004). Figure 2 illustrates a $10 \mathrm{~s}$ segment of ongoing EEG data that has been corrected for ocular artifacts using ICA procedures. The blinks in the data have been identified by the algorithm, and any blink activity deemed statistically independent of the brain activity has been partialled out of the EEG trace. ICA methods provide a more realistic outcome of ocular correction because they take into account the statistical independence of the varying components. Running this algorithm can, however, become quite time-consuming 
and does require a substantial amount of both EEG data and electrode sites. The algorithm will not be able to properly determine the localization and independence of components if too few data or electrode placements are provided.

\section{Segmentation \& Baseline correction}

Segmentation. Once initial filtering and correction methods have been applied, ${ }^{1}$ the EEG is then segmented into discrete "epochs" typically beginning before a stimulus is presented and extending for a period of time after it. An epoch is created for every single "trial" (i.e., every stimulus presentation). If 1000 stimuli had been presented, then 1000 different epochs would now be created. These will also need to be sorted for every stimulus and response category. Suppose the researcher uses an oddball paradigm in which 500 stimuli were presented, 400 of these being frequently occurring "standards" and then at odd times (the remaining 100 trials), a "target" being presented that the subject is asked to detect by pressing a button. The single trial epochs are thus sorted into 400 standards and 100 targets. The targets may further be sub-divided on the basis of performance into targets that were correctly detected ("hits") and those that were not ("misses"). Obviously, the cognitive processes involved in a hit and a miss may be very different. How does the researcher determine the duration of the single trial epoch (also called the "sweep time")? The epoch should include EEG activity prior to the onset of the stimulus in addition to the activity following its presentation. The pre-stimulus period serves as a "baseline" from which the amplitude of the ERPs of interest will be measured. This is because prior to the stimulus, there should be no EEG activity that is related to the stimulus. In actual

${ }^{1}$ It is also possible to filter following segmentation of the single trials and the resulting averaged ERPs should be identical. The filtering of the single trials will be more rapid because fewer data points are involved in the computation. However, with the use of powerful, modern computers, this time difference is incidental. Most researchers therefore prefer to filter the raw, continuous EEG and doing so does have advantages. Attenuating high frequencies by initially filtering the continuous EEG better enables the researcher to visually identify intervals in which the EEG is obviously "bad". Moreover, ICA eye correction methods best operate on data in which very noisy intervals in the EEG have already been excluded. fact, there may be some pre-stimulus activity related to processes such as expectation or prediction of occurrence of the upcoming stimulus. There may also be activity related to the processing of the previous stimulus. The length of the post-stimulus epoch will depend on the cognitive processes of interest. Certain ERP components will have very short latencies, while others may occur much later. It is therefore important to select an appropriate epoch length to ensure that the ERP component of interest is not excluded from the segment. A general rule-of-thumb used by ERP researchers is that the epoch should extend two to three times the period of the major peak of interest. For example, the P3 ERP component is a positive-going ERP component peaking at about $300 \mathrm{~ms}$ after the onset of a target. This peak may occur earlier or much later in different subjects. Therefore, in the case of the P3, the recording epoch should extend to at least 800 to 1000 $\mathrm{ms}$ following stimulus onset. Another means to determine the poststimulus epoch length relies on the actual behavioural response made by the subject following the stimulus, assuming that it is recorded. The response time (RT), the delay between stimulus onset and the response, will vary from trial-to-trial. In this case, the post-stimulus epoch should also be very liberal and extend well beyond the mean RT. Thus, in the example above, if the mean RT were $450 \mathrm{~ms}$, the post-stimulus epoch might be set to be $1000 \mathrm{~ms}$. Also, it is essential that the recording epoch be long enough to allow the ERP activity to return to baseline. Again, because the data are analyzed off-line, the duration of the epoch can always be altered in a subsequent analysis.

The segmentation procedure is illustrated in Figure 3. The Figure displays $10 \mathrm{~s}$ of EEG activity during which time a stimulus (S1) is presented 3 times. The EEG is thus segmented into epochs for each stimulus presentation. In this Figure, the shaded epoch begins $100 \mathrm{~ms}$ prior to the onset of S1 and continues for 900 ms following it. The total epoch thus lasts for $1 \mathrm{~s}$. An ERP is elicited by each stimulus presentation but the amplitude of this response is very small relative to the ongoing EEG. Signal averaging techniques (described below) will be required to allow the researcher to visualize the minute response that is embedded in the much larger amplitude EEG.

Baseline correction. The average of all activity prior to the onset of the stimulus is used to compute a zero voltage pre-stimulus baseline. The pre-stimulus baseline is not necessarily identical to the voltage of the 


\section{Segmenting the "Raw" EEG}

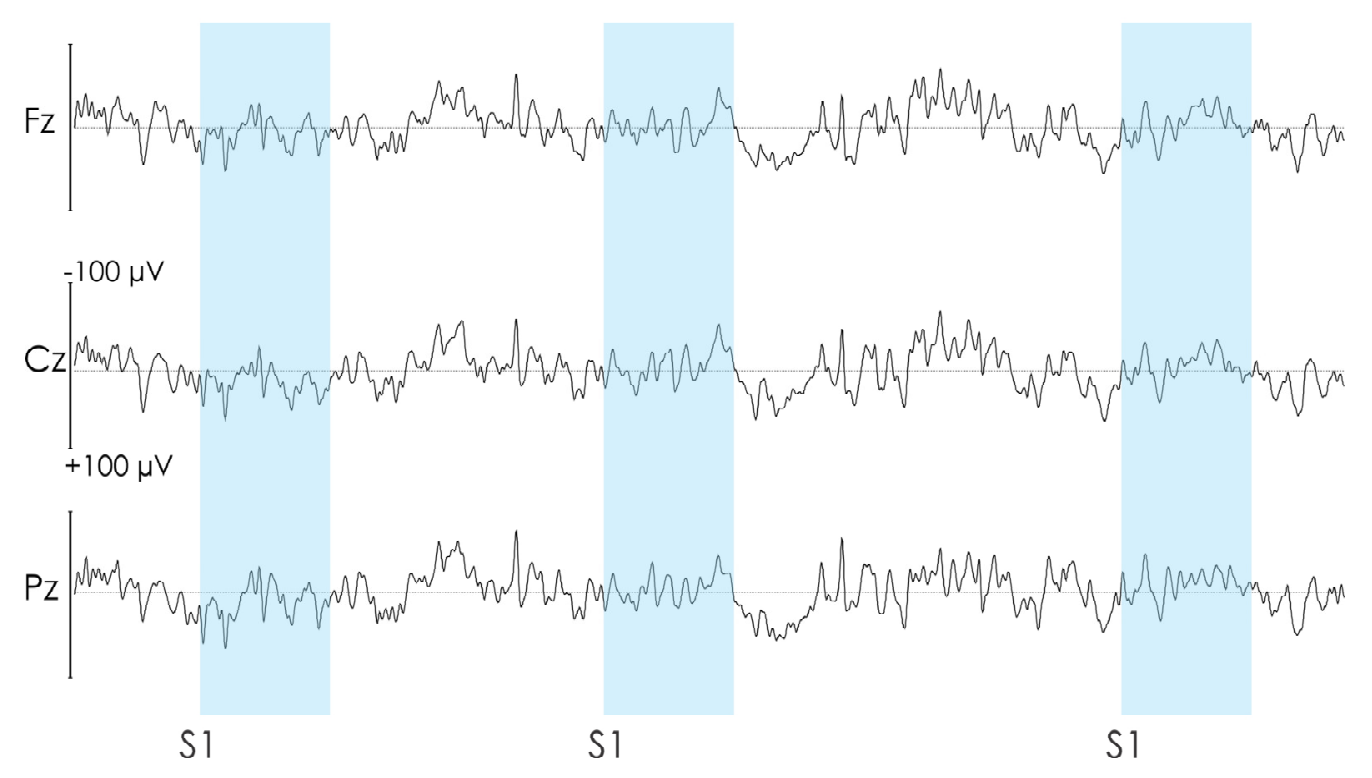

Figure 3 - Segmenting the "raw" EEG. A 10 s display of the "raw", continuous EEG recorded from electrode sites, Fz, $\mathrm{Cz}$ and Pz is illustrated. A stimulus (S1) was presented 3 times during this $10 \mathrm{~s}$ period and the raw EEG is thus cut into 3 different single trial segments or epochs (shaded). Each epoch begins $100 \mathrm{~ms}$ before the onset of S1 and continues for $900 \mathrm{~ms}$ after the stimulus (i.e., a $1000 \mathrm{~ms}$ total duration epoch). An ERP is elicited following presentation of S1 but its amplitude is minute (about $5 \mu \mathrm{V}$ ) and very difficult to observe because it is embedded in the much higher amplitude ongoing raw EEG (about 25-50 $\mu \mathrm{V}$ ). The "signal" is thus buried in the "noise". Signal averaging procedures will be required to enhance the signal-to-noise ratio, allowing the ERP to emerge.

ground electrode (i.e., zero Volts). If the EEG slowly drifts away from the true zero level, the pre-stimulus baseline will also drift in this direction (i.e., not be recorded as a true 0 voltage). This drifting in a negative and positive direction, therefore, needs to be corrected. It could, of course, be corrected by changing the low frequency, time constant. However, the drift may represent a true, slow potential ERP component. In such cases, baseline correction can be used. The algorithm is quite simple and therefore very rapid to compute. The average of all pre-stimulus activity is computed. In Figure 4, the baseline is drawn through the true zero voltage (the ground voltage). The prestimulus activity on this trial is however "above" this baseline; it has drifted in a positive direction. The average of this pre-stimulus activity is about $+5 \mu \mathrm{V}$. The $+5 \mu \mathrm{V}$ average baseline is then subtracted from every data point in the pre and post-stimulus interval. This procedure is then subsequently repeated for every epoch.

\section{Artifact rejection}

As previously indicated, several types of artifacts can contaminate the EEG. Among these, the most prevalent types of artifact include blinks, eye movements, muscle activity, and heartbeats. These artifacts are typically very large compared to an ERP signal and do not reflect true brain activity. Some of these artifacts, as has also been noted, can be corrected through filtering and correction procedures. Other artifact may still remain.

The artifact rejection process involves the identification and rejection of epochs contaminated by artifact. Again, what constitutes an artifact must be defined. This is usually done by stating that the waking EEG should rarely exceed an arbitrary level, for example. Thus, if any of single trial epochs contain EEG activity that exceeds $+100 \mu \mathrm{V}$, it would be it rejected and removed from further analysis. Assuming that the EEG has been filtered to attenuate high and low frequency noise and then corrected for common EOG artifact and pre-stimulus baseline drift, the loss of data due to rejection techniques should be minimal. The selection of the actual criterion of artifact detection, while arbitrary, must be sufficiently sensitive in order to eliminate all trials in which artifact occurs. However, it must also be liberal enough to avoid false alarms and 


\section{Baseline Correction Procedure}

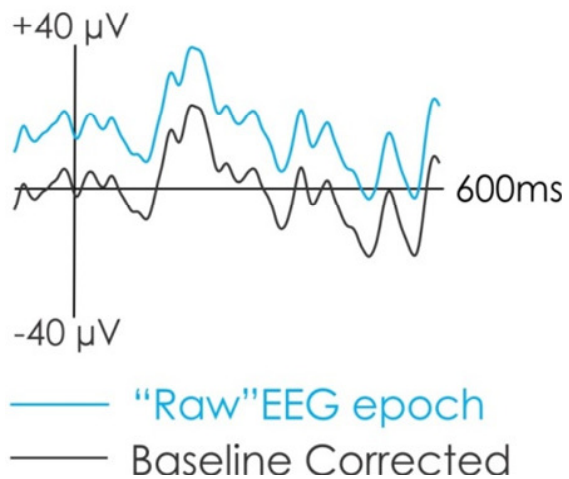

Figure 4 - Baseline correction procedure. A $700 \mathrm{~ms}$, single trial epoch from the $\mathrm{Cz}$ electrode is displayed. The average of all activity in the $100 \mathrm{~ms}$ interval prior to the stimulus is used as a baseline. The Figure illustrates the single epoch before (in blue) and after (in black) baseline correction. The baseline is drawn through a true electronic zero volt voltage. Prior to baseline correction, the pre-stimulus activity on this trial is almost $+40 \mu \mathrm{V}$ "above" zero. The average activity in this pre-stimulus period is then subtracted from every data point in the post-stimulus interval.

an excessive loss of data. Thus, some compromise is inevitable.

\section{Signal Averaging}

Signal averaging is a procedure in which a constant signal (the ERP component of interest) occurring in repeated trials emerges from the random, ongoing background EEG, or noise within these trials. In most cases, the amplitude of the ERP is very small while the amplitude of the ongoing EEG can be very large. Even when filtering and artifact rejection methods have been applied to attenuate noise, ERPs will almost always be difficult to distinguish from the background EEG. Signal averaging techniques are used to reduce the amplitude of the background noise, resulting in an increase in the signal-to-noise (S/N) ratio, allowing the constant signal to emerge.

Certain assumptions must be met for signal averaging techniques to function correctly. The EEG data collected in a single trial is assumed to consist of both the signal (the ERP) and random noise (the ongoing EEG + other artifact). Importantly, the ERP is assumed to be identical (invariant) from one trial to the next. Thus, its amplitude and its peak latency do not vary. Across the trials, the average of an invariant constant is constant (the average of $+5,+5,+5,+5$ across 4 different trials, is of course +5$)$. The noise is, however, assumed to be completely unrelated to the time-locking event (e.g. the stimulus or the response). Its amplitude across the different trials is thus random. It may be positive- or negative-going. Its amplitude might be high or low. The average of a random event over a sufficiently large number of trials should tend towards zero (on the 4 trials above, the noise might measure $+15,-4,-26,+32$, the average being +4.25$)$. With a sufficiently large number of trials, the average of the random events should approach zero allowing the average of the constant signal to emerge.

The amplitude of the random noise decreases as a function of the square root of the number of trials. Thus, after 4 stimulus presentations, the amplitude of the background EEG is reduced by 2 (i.e., it is now $1 / 2$ of its single trial amplitude). Therefore, if the researcher wishes to halve the amplitude of the noise (thus doubling the $\mathrm{S} / \mathrm{N}$ ratio), the number of trials must increase 4 times. If after 16 trials, the $\mathrm{S} / \mathrm{N}$ is not sufficient to allow the signal to emerge and the researchers wants to double it (make the signal twice as large as the noise), 64 trial presentations will now be needed, not 32. And, if that is still not sufficient, 256 trials will be needed to again double the $\mathrm{S} / \mathrm{N}$ ratio and not 128. Figure 5 illustrates the averaging procedure. The upper left portion of the figure illustrates 4 unaveraged superimposed single (or "raw") trials. These single trials contain both background noise and the signal of interest. Immediately below the single trials, the average of the 4 trials is presented. A positive component at about $200 \mathrm{~ms}$ is beginning to emerge. Again, to double the $\mathrm{S} / \mathrm{N}$ ratio of the average, 4 times the number of trials (i.e., 16 trials) must be presented. The upper middle portion superimposes 4 averages of 4 trials each. The average of these 16 trials is presented below the superimposed averages. Now, both the negative and positive components are beginning to emerge from the background noise. To double this $\mathrm{S} / \mathrm{N}$ ratio, 64 trials must be presented. The upper right hand portion superimposes again 4 averages, but each trace is now the average of 16 trials. On the bottom right portion of the Figure, the average of the 64 trials has been computed. The ERP components of interest can be viewed much more clearly here; a negative-going peak at about $100 \mathrm{~ms}$ and a positive peak at about $200 \mathrm{~ms}$. Compare this average to the upper left portion of the Figure in which 4 single trials are superimposed in which the ERPs of interest are embedded in the much higher amplitude background noise. 


\section{Theory of Signal Averaging}

\section{4 single epochs}

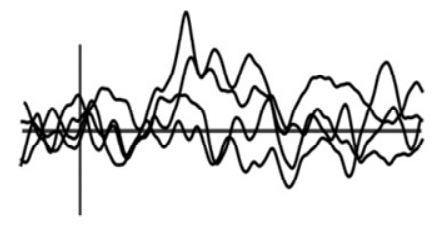

Average of 4

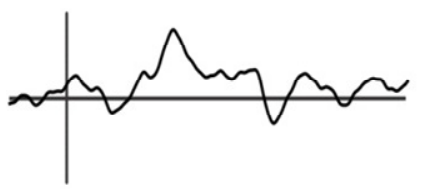

Averages of 4

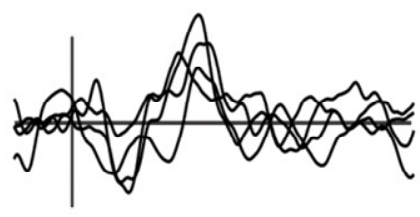

Average of 16

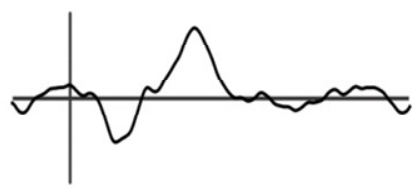

Averages of 16

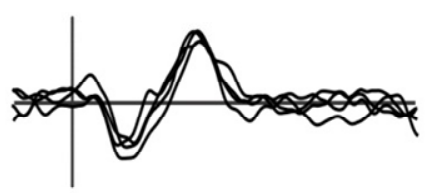

Average of 64

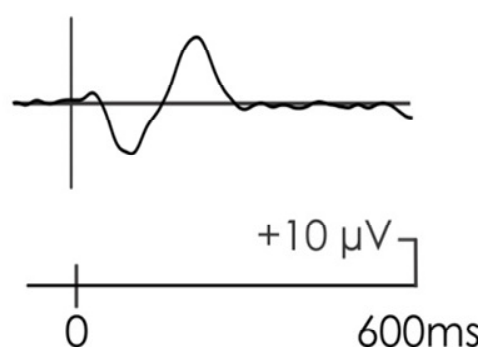

Figure 5 - Theory of signal averaging. Again, a $700 \mathrm{~ms}$ epoch is illustrated beginning $100 \mathrm{~ms}$ prior to stimulus onset. The upper left portion superimposes 4 unaveraged (i.e., single trial)epochs. The small amplitude ERPs are buried in the background noise of the EEG. Averaging procedures are subsequently used to reduce the amplitude of this noise. Immediately below, the average of the 4 trials is presented. This should reduce the amplitude of the background noise by 2 (i.e., the amplitude of the noise should be halved). A small amplitude negativity at about $100 \mathrm{~ms}$ and a larger positive component at about $200 \mathrm{~ms}$ are beginning to emerge from the noise. The upper middle portion superimposes 4 averages of 4 trials each. The average of these 16 trials is presented immediately below this. This should again reduce the amplitude of the noise by 2 compared to when the average was based on 4 trials. Now, the negative and positive components are quite apparent. The upper right hand portion superimposes again 4 averages, but each trace is now the average of 16 trials. On the bottom right portion of the Figure, the average of the 64 trials has been computed. The ERP waveform is now clearly apparent against what is now only small amplitude noise.

While signal-averaging techniques provide an exceedingly powerful means for low amplitude ERPs to emerge in very high amplitude background EEG, there is a cost. As can be observed, the number of stimulus repetitions may have to be exceedingly large. This can be rather discouraging for the researcher and of course, can be very time-consuming. To reduce the number of stimulus presentations that will be required and still ensure a high $\mathrm{S} / \mathrm{N}$ ratio, either the amplitude of the signal must be high or the amplitude of the noise must be low. Although the researcher does not have any control over the amplitude of the signal, they do have some control over the amplitude of the background EEG. It is often much easier to obtain high quality data by decreasing possible sources of noise during the collection of the raw data, rather than increasing the number of trials. To reiterate, priority must be given to obtaining the best possible clean, artifact (noise) free raw data. Filtering, correction of artifact and signal averaging techniques will reduce the amplitude of the background noise of the raw data. It is much better however to minimize sources of noise during the actual recording of the EEG.

\section{Quantifying ERPs}

The final step in an ERP experiment is the measurement and quantification of the different ERP components, and applying these measurements to statistical analyses. Most ERP experiments compute the amplitude and latency of the various components. This section will discuss the quantification of these components using different measurement techniques, 
Table 1 - A comparison of peak amplitude and mean amplitude measurement techniques. An interval of 5 data points have been "cut out" from the averaged ERP waveform. The data for 3 subjects appear in each row. The maximum peak is identified in italics. The grand average, calculated by averaging data points $1,2 \ldots 5$ of the 3 subjects appears in the last row. The mean amplitude of the 5 data appears in the right column for the 3 subjects. The mean of this column (i.e., the mean of the mean amplitudes is identical to the mean of the 5 data points within the grand average). On the other hand, the mean of the peak amplitudes does not equal the mean of the data points within the grand average.

\begin{tabular}{ccccccc}
\hline Subject & 1 & 2 & 3 & 4 & 5 & Mean \\
\hline 1 & 7.0 & 9.0 & 11.0 & 10.0 & 8.0 & 9.0 \\
2 & 6.0 & 9.0 & 7.0 & 7.0 & 3.0 & 6.4 \\
3 & 11.0 & 14.0 & 12.0 & 15.0 & 10.0 & 12.4 \\
Grand Average & 8.0 & 10.7 & 10.0 & 10.7 & 7.0 & 9.3 \\
\hline
\end{tabular}

and the statistical analysis options available. An important warning: the terms peaks and components are often interchangeably used when discussing ERP waveforms. Peaks and components are not the same. The point at which the voltage reaches a maximum value (the "peak") does not necessarily reflect an ERP component. Certain factors such as noise and importantly, other and overlapping components, can influence the maximum voltage in a waveform.

Baseline-to-Peak Measurement. Two common methods are used to measure the amplitude of an ERP component. Most labs now use a baseline-to-peak measure. Thus, the average of all activity in the prestimulus period is used as a zero voltage baseline from which the various components are measured. Some labs also use a peak-to-peak measure (the trough of one peak is measured relative to the trough of the next peak) but if there is an experimental effect, it is difficult to know if this outcome is a result of an effect on the first or second peak.

Peak Amplitude Measurement. The first quantification method, peak amplitude measure, finds the maximum amplitude of a peak in a predetermined window of time for each individual subject. Thus, an auditory "N1" ERP component usually occurs at about $100 \mathrm{~ms}$ following stimulus presentation. It might thus be defined and quantified as the maximum negative peak occurring between 60 and 140 ms. This method can be problematic in that the maximum amplitude in a certain time interval may not reflect the actual peak of an ERP component (Luck, 2014; Picton et al., 2000). For example, there may be several peaks within a particular time window and some of these peaks will be remaining unaveraged noise. Peak amplitude measures will arbitrarily measure the largest of these peaks, regardless of whether this truly reflects the actual ERP component of interest or the ERP component + noise. The mean of all subjects' maximum peak amplitude will inevitably be larger than the maximum peak that is apparent in the grand average (again, the average of all subjects' averages). See the section below describing the alternate, mean amplitude measurement technique, for additional discussion of this issue.

Mean Amplitude Measurement. A second method, mean amplitude measure, calculates the mean voltage of the waveform in a predetermined window of time. This method is often applied when there is no single distinct peak for an ERP component of interest within the time interval (Duncan et al., 2009; Luck, 2014; Picton et al., 2000). It is also based on the fact that cognitive processes do not occur at a definitive point in time with perhaps millisecond precision. This is implicitly what the peak amplitude measurement however assumes. Rather a cognitive process occurs over several milliseconds and thus the averaging of all data within the pre-defined time period would be more appropriate. Another advantage of this method is that it is less sensitive to high-frequency noise or multi-peak intervals because the average of a range of data points is used rather than a single time point. The mean amplitude measure thus effectively acts as a type of high filter, by averaging together rapidly alternating negative- and positive-going peaks. A major problem with the maximum peak detection method is that background noise will often contribute to the peak in question. Averaging over several peaks and valleys overcomes this problem. The mean amplitude measurement technique is also a linear measure. As such, the mean amplitude of a component averaged across all subjects is equal to the mean of this component in the grand average (Luck, 2014). This is illustrated in Table 1 . In this Table, an interval containing 5 hypothetical data points is presented for 3 subjects. On the bottom of the Table, the grand average of these 3 subjects within the 5 data point interval is computed (i.e., the average of data point 1 , the average 
of data point 2 ...the average of data point 5 for the 3subjects). In the right-column the mean peak detection method averages the amplitude of the 5 data points for the 3 subjects. The mean of the mean amplitude measures for the 3 subjects is identical to the mean of the 5 data points in the grand average. On the other hand, the maximum peak among the 5 data points is 11,9 and $15 \mu \mathrm{V}$ for subjects 1,2 and 3 respectively. Thus, the mean of the maximum peaks (11.7) of the 3 subjects overestimates the peak in the grand average. The use of the mean amplitude measurement technique thus makes it possible to directly compare the grandaveraged ERP waveforms to the mean of this component in the statistical analyses. This is not the case when peak amplitude measurement techniques are used.

There are also different methods to determine the time interval within which the mean amplitude of the component of interest is measured. One method is based on existing literature, such as the $60-140 \mathrm{~ms}$ time interval window used for the $\mathrm{N} 1$ example above. Thus, for each subject, N1 would be measured as the mean amplitude of all data points within the $60-140 \mathrm{~ms}$ interval in each individual subject. Another method uses the grand average (again, the average of all subjects' average). Because the grand average should contain very little noise, the peak of the component of interest should be easily identifiable. Again, in the example of $\mathrm{N} 1$, perhaps $\mathrm{N} 1$ reaches a maximum amplitude (or "peaks") at $100 \mathrm{~ms}$ in the grand average. $\mathrm{N} 1$ might then be measured as the mean of all data points within +25 ms of this peak (i.e., from 85 to 135 $\mathrm{ms}$ ) in individual subjects.

Latency Measurement. ERP latencies are usually measured at the time of when a peak reaches its maximum amplitude (Duncan et al., 2009; Luck, 2014; Picton et al., 2000). Hence, peak latency techniques are commonly paired with peak amplitude measures. All of the disadvantages associated with peak amplitude measures also apply with peak latency measures. Most notably, if there are multiple peaks within a time window, or if the peak of a component is rather flat, the maximal voltage in that time window may not reflect a true measure of the ERP component's latency

Another method, fractional area latency, is analogous to mean amplitude measures. This technique computes the area under the ERP waveform over a predetermined time interval and then finds the time point that divides this area into a predetermined fraction (Hansen \& Hillyard, 1980). Commonly, the fraction will be a half; therefore, the time point that divides the area under the curve in half is deemed the latency of the ERP component (Luck, 2014). This technique is not appropriate for measuring the absolute latency of a component, or if overlapping ERP components are present within the same time window. Measuring the latency of ERP components can thus be extremely difficult as all methods have multiple drawbacks. Therefore, whichever technique better suits the experimental design of an ERP study should be used. The method used must be clearly specified and extreme caution must be applied in interpreting these results.

Topographical maps. Multi-channel ERPs allow for the display of cognitive activity as it varies over time (the temporal dimension) and as it varies across the scalp (spatial dimension). Variations in ERPs across the scalp are usually displayed as multiple waveforms as a function of time at the different electrode locations. The previous Figures in this article use this convention. However, the spatial aspects of the various ERPs, where the component is maximum and where it is minimum in amplitude, are not easily visualized in this method. The spatial dimension of ERP components is better displayed using voltage distribution maps. Various kinds of maps can be computed and displayed. Of course, there are no actual data between electrode sites. Voltage maps are however made to be continuous by interpolating values between electrode sites where actual data exist. Thus, let us assume that a component measures $8 \mu \mathrm{V}$ at $\mathrm{Fz}$ and $4 \mu \mathrm{V}$ at $\mathrm{Cz}$. One could interpolate the value for $\mathrm{FCz}$ (half way between $\mathrm{Fz}$ and $\mathrm{Cz})$ to be $6 \mu \mathrm{V}$. Similarly, the value for a site half way between $\mathrm{FCz}$ and $\mathrm{Cz}$ could also be interpolated to be 5 $\mu \mathrm{V}(6+4 \mu \mathrm{V} / 2)$. Interpolations of data do need to take into account other "nearest neighbours", for example, electrodes located laterally, and weigh their values accordingly. The upper portion of Figure 6 provides a 64-channel recording of an auditory ERP. The reference site was the tip of the nose. A brief duration, moderate intensity binaural auditory stimulus was presented every $2 \mathrm{~s}$. As can be seen, a large negativity (the N1) is apparent at about $100 \mathrm{~ms}$. An enlargement of the $\mathrm{Fz}, \mathrm{Cz}$ and M2 electrodes is illustrated on the right portion of the Figure. N1 is maximum in amplitude overfrontal and central sites of the scalp. At inferior and lateral sites such as M1 and M2 (or TP9, TP10, the left and right mastoids), N1 inverts in polarity and is recorded as positive-going. In the lower, right portion of the Figure, a topographic map of the scalp distribution of 


\section{Scalp Distribution Mapping}
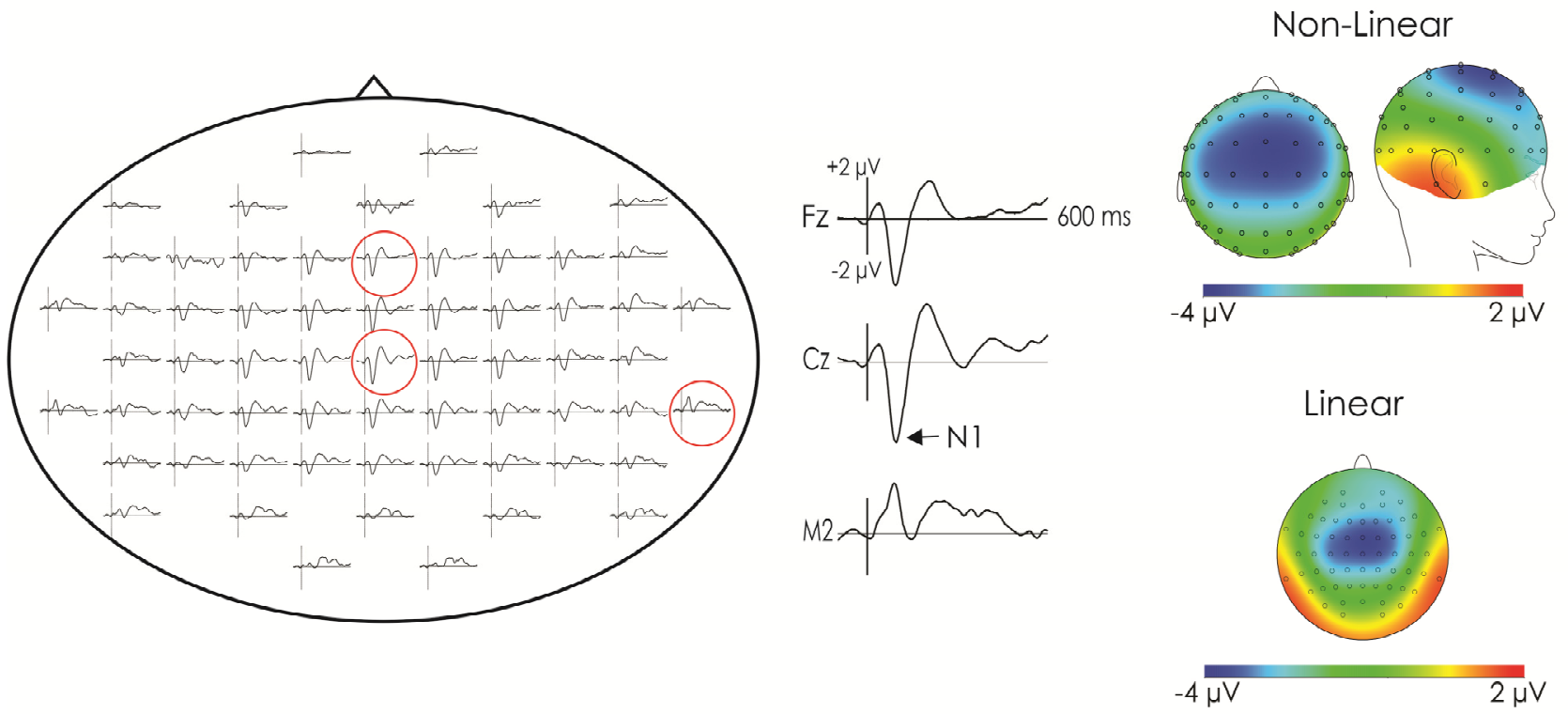

Figure 6 - Scalp distribution mapping. Left portion: a 64-multichannel recording. The reference site was the tip of the nose. The ERP was elicited by a $200 \mathrm{~ms}$ moderate intensity auditory stimuli presented every $2 \mathrm{~s}$. The waveforms are "grand" averages based on 8 subjects (the average of the 8 subjects' averages). To the right of the 64 channel array, a zoom of the Fz, Cz and M2 electrodes is presented. The downward deflection at about $100 \mathrm{~ms}$ is the auditory N1. It was largest in amplitude over fronto-central areas of the scalp. At sites inferior to the Sylvain fissure (e.g., M1 and M2), N1 inverts in polarity and is recorded as a positive-going component. The scalp distribution of N1 is best displayed in a map. These are illustrated to the right of the Figure. A spline-interpolated algorithm was used to compute a continuous topographic map of N1. Nonlinear and linear views are illustrated. The non-linear view provides a visualization of the electrodes as would be observed by the human eye from, in this example, the top and the side of the head. Thus, in the top view, electrodes over the lateral and inferior scalp are closely spaced. In the side view, electrodes near the top of the head are closely spaced. In the linear top view, electrodes are evenly spaced and the projection continues farther down the scalp to include very inferior FT9 and FT10 and the two mastoid (TP9 and TP10) electrode sites. In both nonlinear and linear views, the map indicates that the amplitude of N1 is maximum over frontocentral areas of the scalp while a polarity inversion (N1 is recorded as a positive potential) is apparent at inferior regions.

N1 is presented. A non-linear and a linear view are provided. The non-linear view presents both a top and side perspective. These linear aspects reflect the views of the head as seen by the human eye. Thus, in the top view, electrodes at the top of the head (e.g., Cz-C1-C3, $\mathrm{Cz}-\mathrm{C} 2-\mathrm{C} 4)$ are spaced farther apart than electrodes at lateral-inferior regions (e.g., C5-T7-C6-T8). Consequently, in order to obtain a better view of the lateral and inferior electrodes, a lateral, side projection is also required, in this case, the right side of the head. In this case, electrodes near the ears are placed farther apart than electrodes near the top and anteriorposterior portions of the head, again in keeping with how the eye would view the side-to-top of the head. It is also possible to calculate topographic maps with the use of a linear, equidistant perspective. Thus, the distance between the electrodes is equal. In this view, the projection may be extended down $20^{\circ}$ below the Fp1-T7-Oz-T8-Fp2 circumference to show data from the most inferior electrodes. A top, linear equidistance map extended down $20^{\circ}$ is illustrated in the bottom right portion of the Figure.

As mentioned, the simplest maps are computed using a "nearest neighbour" algorithm (Duffy, Burchfiel, \& Lombroso, 1979). Most often, a four nearest neighbours algorithm is used. A serious issue with the nearest neighbours approach is that discontinuities may appear in the maps. Spline interpolations provide for smoothly changing maps. Because the head is spherically shaped, spherical spline maps (Perrin, 
Pernier, Bertrand, \& Echallier, 1989) currently tend to be most often used. The maps in Figure 6 were computed using spline-interpolated maps.

A major issue with the use of maps is that they are often misinterpreted as reflecting underlying sources or "generators". In the Figure above, N1 was maximum over frontal regions of the scalp. Maps of the scalp voltage distribution are not however maps of the sources! Although N1 might be maximum in amplitude over frontal regions, it is not necessarily the frontal regions that were most activated by the auditory stimulus. Indeed, in the case of N1, it has long been known that its fronto-central scalp distribution is probably best explained by the orientation of source dipoles in and around the auditory (not the frontal) cortex (Scherg, Vajsar, Picton, 1989). Scalp distribution maps do not directly provide a map of the areas of the brain activated in a particular condition. Nevertheless, even if the scalp distribution maps cannot be used as maps of the underlying sources, they can be used in a different way. Components whose scalp topographies vary must have different intra-cranial generators. Thus, it also logically follows that if the scalp distribution of an ERP component is different in two conditions, then the underlying cerebral generators must be different in these conditions. It does not logically follow however that if the scalp distribution is the same in two different conditions, then the underlying sources must be the same. It is possible to have identical scalp maps as a result of different source dipole activity (Picton et al., 1995).

Statistical Analyses of Scalp Distribution Data. Statistical analyses of the scalp distributions of the ERP components across conditions or between groups need to be interpreted with caution (Picton, 1988). These analyses are fraught with difficulties. Most of the data in the scalp distribution maps are not "real" data. Rather they are interpolated. In the example above, the researcher did not have any real data for the $\mathrm{FCz}$ placement. Rather it was interpolated using a nearest neighbor computation. Statistical analyses should only be applied to the actual data.

One of the major reasons for collecting data from multiple electrode sites is to determine if an experimental manipulation affects the scalp distribution of an ERP component. This would usually be compared by a 2-way analysis of variance (ANOVA) to examine the interaction between the experimental condition and the scalp distribution. Unfortunately, the ANOVA procedure is poorly suited for this purpose. An assumption for the use of the ANOVA is that the experimental manipulation will result in an additive effect (a constant is added to each variable). The experimental manipulation causes a multiplicative effect on ERP scalp voltages. This issue has been discussed by McCarthy and Wood (1985) and Picton et al. (1995). Again, let us assume that the amplitude of the $\mathrm{N} 1$ component measures $-8 \mu \mathrm{V}$ at the frontal electrodes site (where $\mathrm{N} 1$ is largest) when the subject actively attends to the auditory stimuli. At central sites, it might measure half this amplitude, $-4 \mu \mathrm{V}$, and at parietal sites, $-2 \mu \mathrm{V}$. Note that this is a multiplicative effect (the voltage drops by $1 / 2$ from $\mathrm{Fz}$ to $\mathrm{Cz}$ to $\mathrm{Pz}$ ), not a linear one (the voltage does not drop by $4 \mu \mathrm{V}$ at each equidistant electrode site as one moves in an anterior to posterior direction). Now, let us suppose that in a second "ignore" condition, the amplitude at Fz drops to $-4 \mu \mathrm{V}$. In short, the amplitude decreases by $4 \mu \mathrm{V}$. The additive model of the ANOVA procedure would also examine if the amplitude also decreases by $4 \mu \mathrm{V}$ at both $\mathrm{Cz}$ and $\mathrm{Pz}$. Thus, $\mathrm{N} 1$ would now measure 0 and $+2 \mu \mathrm{V}$ at $\mathrm{Cz}$ and $\mathrm{Pz}$ respectively. If so, this would be reflected in a significant main effect of the experimental condition and a non-significant condition $\mathrm{x}$ electrode site interaction (i.e., the effect is the same across all electrode sites). In reality, the amplitude would tend to be halved (a multiplicative effect) at $\mathrm{Cz}$ and $\mathrm{Pz}$, thus measuring -2 and $-1 \mu \mathrm{V}$ respectively. Thus, the ANOVA might point to a significant condition $\mathrm{x}$ electrode site interaction because the effect was larger at $\mathrm{Fz}$ (a $4 \mu \mathrm{V}$ difference) than at $\mathrm{Pz}$ (a $1 \mu \mathrm{V}$ difference). This would lead to a false interpretation of the experimental effect. The experimental effect had the same, constant multiplicative effect at all electrode sites. The ignoring of the stimuli caused the amplitude of $\mathrm{N} 1$ to be reduced by $50 \%$ at all sites. The presence or absence of a significant interaction cannot therefore be easily interpreted. McCarthy and Wood (1985) have provided a scaling method to normalize the scalp distribution data. Unfortunately, the McCarthy and Wood normalization procedure has also been demonstrated to potentially produce erroneous results and cannot correct for the multiplicative effects on interactions involving an electrode factor (Urbach and Kutas, 2002).

\section{Conclusion}

The two articles in this series have described the realtime recording of the EEG from multiple electrode sites and how these are affected by various cognitive paradigms. The present article discussed the basic 
approaches for the analysis of ERP data. The advent of extremely powerful, but inexpensive, microcomputers and low cost multichannel amplifier systems hasmade the collection of both the performance and physiological data almost exponentially easier than in the past. ERP research has a long history dating back to the pioneers in the 1950s and 1960s. One of the major benefits of modern computer systems is that many complex off-line analyses can now be carried out on the data. An advantage of the off-line approach is that the raw data are not altered. Thus, any analyses that are carried out can later be reversed and modified. The EEG data are typically examined for artifacts. Many sources of artifact can be removed using digital filtering techniques. A common artifact is that caused by eye blinks and movements. This source of contamination is especially problematic over anterior regions of the scalp. Sophisticated algorithms, such as independent component analysis, can be used to correct for eye movement artifact, although the EEG does need to be recorded from a large number of scalp electrodes. The filtered and corrected continuous EEG data then need to be "cut" into segments, or epochs, of varying lengths and sorted according to the different stimuli that have been presented and types of responses made by the subject. Waxing and waning of the EEG within the epoch can be corrected. Epochs in which the amplitude of the EEG is unusually large are often rejected. The amplitude of the actual change in the electrical activity that is elicited by the physical stimulus or cognitive task demands (i.e., the ERP signal) is usually much smaller than the ongoing EEG in which it is embedded. Signal averaging techniques have long been employed to reduce the amplitude of the background noise, allowing the ERP signal to emerge. If the amplitude of the ERP is very small and the amplitude of the ongoing EEG is very large, many stimulus repetitions will probably be required and the time required to collect the data may thus be quite long.

With the arrival of modern imaging techniques such as PET and the FMRI, it was thought that ERP research would fade away unable to compete with the spatial resolution capabilities of these (much more expensive) systems. As discussed in this article, it is possible to map the scalp distribution of the different ERP components. Nevertheless, the scalp distribution maps should not be interpreted to be maps of the underlying intracranial sources. Source localization techniques that rely on the scalp distribution maps can be used to mathematically compute the possible intracranial dipole sources. Source localization methods do require considerable human expertise and are not fully automated and for this reason, were not discussed in this article. There are several disadvantages to the use of hemodynamic measures imaging systems. Most importantly, their temporal resolution is much slower (in the order of seconds) than that afforded by ERP methods (millisecond resolution). For this reason, if anything, there are now many more ERP researchers than in the past. ERPs have thus become an integral part of the cognitive neuroscientist's toolbox and modern imaging systems can now record ERPs concurrently during the fMRI imaging procedure.

Ease of data collection and analysis is no substitute for carefully designed studies and the collection of high quality raw data. It is best to remove sources of noise and artifact during the actual data collection. In spite of the best efforts of the researcher, subjects will blink and move. Modern systems also allow for the low cost collection of data from many different scalp sites. Even though data are now routinely recorded from 64, 128 and 256 electrode sites, how these multichannel data are to be analyzed is still however much debated.

\section{References}

Belouchrani, A., Abed-Meraim, K., Cardoso, J. F., \& Moulines, E. (1997). A blind source separation technique using second-order statistics. Signal Processing, IEEE Transactions on, 45, 434-444.

Cook, E. W., \& Miller, G. A. (1992). Digital filtering: Background and tutorial for psychophysiologists. Psychophysiology, 29, 350-362.

Dawson. G.D. (1954). A summation technique for the detection of small evoked potentials. Electroencephalography and Clinical Neurophysiology, 6, 65-84

Duffy, F. H., Burchfiel, J. L., \& Lombroso, C. T. (1979). Brain electrical activity mapping (BEAM): a method for extending the clinical utility of EEG and evoked potential data. Annals of Neurology, 5, 309-321.

Duncan, C. C., Barry, R. J., Connolly, J. F., Fischer, C., Michie, P. T., Näätänen, R., ... \& Van Petten, C. (2009). Event-related potentials in clinical research: guidelines for eliciting, recording, and quantifying mismatch negativity, P300, and N400. Clinical Neurophysiology, 120, 1883-1908.

Gratton, G., Coles, M. G., \& Donchin, E. (1983). A new method for off-line removal of ocular artifact. Electroencephalography and Clinical Neurophysiology, 55, 468-484. 
Hansen, J. C., \& Hillyard, S. A. (1980). Endogeneous brain potentials associated with selective auditory attention. Electroencephalography and Clinical Neurophysiology, 49, 277-290.

Joyce, C. A., Gorodnitsky, I. F., \& Kutas, M. (2004). Automatic removal of eye movement and blink artifacts from EEG data using blind component separation. Psychophysiology, 41, 313-325.

Jung, T. P., Makeig, S., Humphries, C., Lee, T. W., Mckeown, M. J., Iragui, V., \& Sejnowski, T. J. (2000). Removing electroencephalographic artifacts by blind source separation. Psychophysiology, 37, 163178.

Jung, T. P., Makeig, S., Westerfield, M., Townsend, J., Courchesne, E., \& Sejnowski, T. J. (2000). Removal of eye activity artifacts from visual event-related potentials in normal and clinical subjects. Clinical Neurophysiology, 111, 1745-1758.

Lins, O. G., Picton, T. W., Berg, P., \& Scherg, M. (1993). Ocular artifacts in EEG and eventrelated potentials I: Scalp topography. Brain Topography, 6, 51-63.

Lins, O. G., Picton, T. W., Berg, P., \& Scherg, M. (1993). Ocular artifacts in recording EEGs and event-related potentials II: source dipoles and source components. Brain Topography, 6, 65-78.

Luck, S. J. (2014). An introduction to the event-related potential technique. MIT press.

Luck, S. J. (2005). An Introduction to the Event-Related Potential Technique. MIT press.

McCarthy, G., \& Wood, C. C. (1985). Scalp distributions of event-related potentials: an ambiguity associated with analysis of variance models. Electroencephalography and Clinical Neurophysiology/Evoked Potentials Section, 62, 203-208.

Oostenveld, R., \& Praamstra, P. (2001). The five percent electrode system for highresolution EEG and ERP measurements. Clinical Neurophysiology, 112, 713719.

Perrin, F., Pernier, J., Bertrand, 0., \& Echallier, J. F.
(1989). Spherical splines for scalp potential and current density mapping. Electroencephalography and Clinical Neurophysiology, 72, 184-187.

Picton, T.W., Lins, O.G., \& Scherg, M. (1995). The recording and analysis of event-related potentials. The Handbook of Neuropsychology, 10, 3-73.

Picton, T. W. (2011). Human auditory evoked potentials. Plural Pub Incorporated, San Diego, CA.

Picton, T.W. (1988). The mapping of the brain. Clinical Evoked Potentials, 5, 2-10.

Picton, T., Bentin, S., Berg, P., Donchin, E., Hillyard, S., Johnson, R., . . Rugg, M. (2000). Guidelines for using human event-related potentials to study cognition: Recording standards and publication criteria. Psychophysiology, 37, 127-152.

Picton, T. W., Linden, R. D., Hamel, G., \& Maru, J. T. (2008). Aspects of averaging. Paper presented at the Seminars in Hearing.

Scherg, M., Vajsar, J., Picton, T.W. (1989). A source analysis of the late human auditory evoked potentials. Journal of Cognitive Neuroscience, 1, 336-355.

Tavakoli, P. \& Campbell, K. (2015). The recording and quantification of event-related potentials: I. Stimulus presentation and data acquisition. The Quantitative Methods for Psychology, 12, 89-97.

Urbach, T. P., \& Kutas, M. (2002). The intractability of scaling scalp distributions to infer neuroelectric sources. Psychophysiology, 39, 791-808.

VanRullen, R. (2011). Four common conceptual fallacies in mapping the time course of recognition. Perception Science, 2, 365-370.

Widmann, A., \& Schröger, E. (2012). Filter effects and filter artifacts in the analysis of electrophysiological data. Frontiers in Psychology, 3, 1-5.

Woestenburg, J.C., Verbaten, M.N., Slangen, J.L. (1983). The removal of the eye-movement artifact from the EEG by regression analysis in the frequency domain. Biological Psychology, 16, 127-147.

\section{Citation}

Tavakoli, P., \& Campbell, K. (2015). The Recording and Quantification of Event-Related Potentials: II. Signal Processing and Analysis. The Quantitative Methods for Psychology, 11(2), 98-112. 